\title{
Probing the Geometry of Supernovae with Spectropolarimetry
}

\author{
Douglas C. Leonard, Alexei V. Filippenko, and Thomas Matheson \\ Department of Astronomy, University of California at Berkeley, Berkeley, CA 94720-3411
}

\begin{abstract}
We present results from a spectropolarimetric survey of young supernovae completed at the Keck Observatory, including at least one example from each of the major supernova types: Ia (1997dt), Ib (1998T, 1997dq), Ib/c-pec (1997ef), IIn (1997eg), and II-P (1997ds). All objects show evidence for intrinsic polarization, suggesting that asphericity may be a common feature in young supernova atmospheres.
\end{abstract}

\section{INTRODUCTION}

Are supernovae (SNe) round? This simple question belies a menacing observational challenge, since all extragalactic SNe remain unresolvable point sources throughout the crucial early phases of their evolution. Since a hot young supernova (SN) atmosphere is dominated by electron scattering, which, by its nature, is highly polarizing, a powerful tool for investigating SN geometry is spectropolarimetry of the expanding fireball shortly after the explosion (Fig. 1).

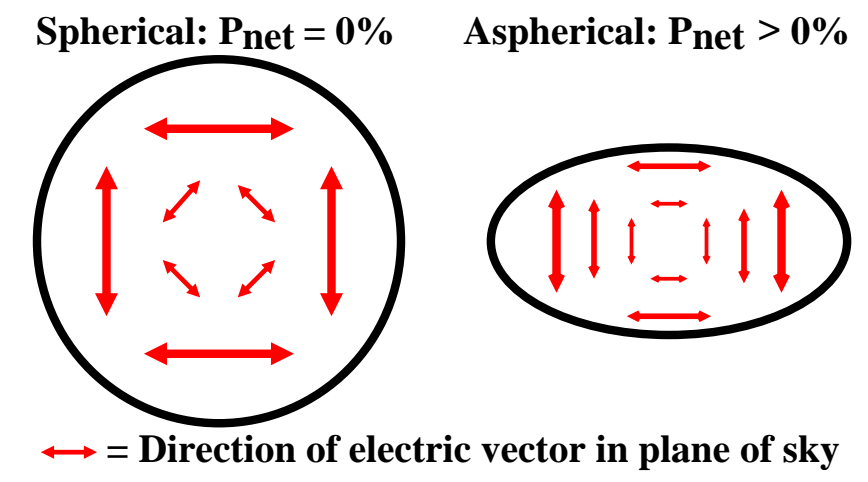

FIGURE 1. Polarization magnitude and direction in the plane of the sky for resolved electron-scattering atmospheres; for an unresolved source (i.e., a SN), only the net magnitude and direction can be measured. An aspherical source produces a net linear polarization due to incomplete cancellation of the directional components of the electric vectors. Note that the more highly polarized light (longer arrows) comes from the limb regions in this simple model. 
Typical polarizations of $\sim 1 \%$ are expected for moderate $(\sim 20 \%)$ SN asphericity [1]. Detecting such low polarization requires a very high signal-to-noise ratio, which has limited previous detailed spectropolarimetric studies to only the two brightest recent events, SN 1987A [2] and SN 1993J [3,4]. We thus began a program to obtain spectropolarimetry of nearby SNe using the 10-m Keck telescopes.

A complication in the interpretation of all polarization measurements is disentangling the polarization intrinsic to the object from interstellar polarization (ISP) produced by dust along the line-of-sight. Fortunately, the ISP is constant with time and a smoothly varying function of wavelength. Therefore, we consider distinct spectral polarization features, temporal changes in the overall polarization level, or continuum polarization characteristics differing from the known form produced by interstellar dust as evidence for intrinsic SN polarization.

\section{RESULTS AND DISCUSSION}

Single-epoch polarization data for six SNe of various types are shown in Fig. 2. Since determining the intrinsic SN polarization level requires knowledge of the (unknown) ISP, we focus instead on the sharp changes seen in the polarization at the location of strong features in the total flux spectra; these features remain, regardless of the ISP contribution. Since all the objects studied possess spectropolarimetric line features, we conclude that all types of SNe show evidence for intrinsic polarization at early times, suggesting that asphericity may be a ubiquitous SN characteristic.

The fact that the strongest spectropolarimetric features are often seen in the troughs of strong P-Cygni lines is not surprising. A simple explanation may be that P-Cygni absorption selectively blocks photons coming from the central, more

forward-scattered (and thus less polarized) regions, thereby enhancing the relative contribution of the more highly polarized photons from the limb regions (c.f., Fig. 1). Unfortunately, since different (allowable) choices for the ISP can make inferred intrinsic polarization dips become peaks and vice-versa [5], we cannot say for certain whether the changes seen here in P-Cygni troughs represent increases or decreases in the intrinsic polarization level. We do note, however, that trough polarization increases are seen in the ISP-corrected data of both SN 1987A [2] and SN 1993J $[4]$.

A total flux spectrum dominated by strong line emission without P-Cygni absorption is a distinguishing characteristic of SNe IIn [6], likely resulting from an intense interaction between the SN and a dense circumstellar environment (CSM). SN 1997eg (Fig. 2) shows sharp polarization changes across its strong, multicomponent emission lines, suggesting distinct scattering origins for the intermediate (full width at half maximum $(\mathrm{FWHM}) \approx 2000 \mathrm{~km} / \mathrm{s}$ ) and broad $(\mathrm{FWHM} \approx$ $15000 \mathrm{~km} / \mathrm{s}$ ) components. Two additional spectropolarimetric epochs (not shown) revealed a change in continuum polarization level of $\sim 1 \%$ over 78 days, further confirming the presence of intrinsic polarization. A detailed analysis combining 


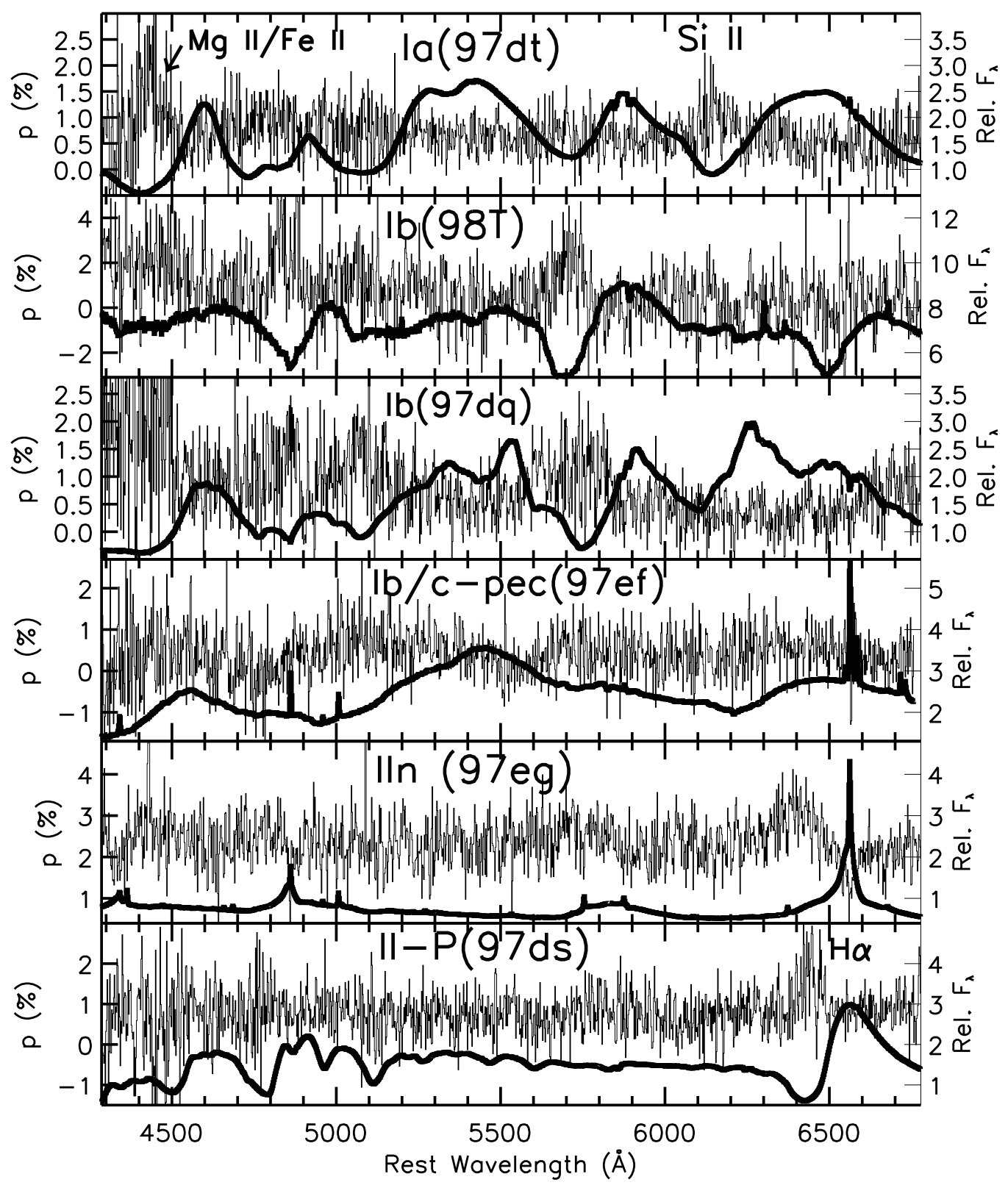

FIGURE 2. Spectropolarimetry (thin lines) and total flux spectra (thick lines) of nearby SNe, all within two months of the explosion. The recession velocities of the host galaxies have been removed for all objects. Note that arbitrary, but allowable (from reddening considerations), estimates of the ISP have been removed from the polarizations of SNe 1997dt, 1998T, 1997dq, and $1997 \mathrm{ds}$ in order to better highlight the line features. Since the ISP is in general not known, the intrinsic continuum polarization levels are uncertain; spectropolarimetric line features, however, exist regardless of the ISP, indicating polarization intrinsic to all of these SNe. 
spectropolarimetry and total flux spectra of another IIn event, SN 1998S, also found evidence for a highly aspherical ( $~ 45 \%$ ) continuum scattering region, with the CSM likely distributed in a disk-like or ring-like morphology, quite similar to what is seen directly in SN 1987A [7], except much closer to the progenitor in the case of $1998 \mathrm{~S}$ [5].

\section{CONCLUSION}

The number of SNe studied spectropolarimetrically is still very small, but early indications are that all types reveal intrinsic polarization if examined in sufficient detail. In addition to the implications of spectropolarimetry on the core-collapse mechanism, the mass-loss history of evolved stars, and the spatial distribution of SN ejecta, this work has direct consequences on the use of SNe as cosmological distance indicators. Although the empirically-based, standard-candle technique used to measure SN Ia distances does not rely on spherical symmetry, distances derived to SNe II-P through the "expanding photosphere method" [8] would need to be corrected for directionally-dependent flux if asphericity is found to be common among this SN class. We note that SN 1999em, a type II-P event discovered shortly after this conference, showed no evidence for intrinsic polarization when it was observed less than two weeks after the explosion [9]. It will be interesting to see if it remains unpolarized at an age comparable to the II-P observation (SN $1997 \mathrm{ds}$, observed $\sim 50$ days after explosion) presented here.

\section{ACKNOWLEDGMENTS}

We thank Aaron Barth for useful discussions and assistance with the observations

and data reduction. Supernova research is supported at UC Berkeley through NSF grant AST-9417213 and NASA grant GO-7434.

\section{REFERENCES}

1. Höflich, P. A., Astron. Astrophys. 246, 481 (1991).

2. Jeffery, D. J., Ap. J. 375, 264 (1991).

3. Trammell, S. R., Hines, D. C., and Wheeler, J. C., Ap. J. 414, 21 (1993).

4. Tran, H. D., Filippenko, A. V., Schmidt, G. D., Bjorkman, K. S., Jannuzi, B. T., and Smith, P. S., P.A.S.P. 109, 489 (1997).

5. Leonard, D. C., Filippenko, A. V., Barth, A. J., and Matheson, T., Ap. J. submitted (astro-ph/9908040).

6. Schlegel, E. M., M.N.R.A.S. 244, 269 (1990).

7. Crotts, A. P. S., and Heathcote, S. R., Ap. J. in press (astro-ph/9907367).

8. Kirshner, R. P., and Kwan, J., Ap. J. 193, 27 (1974).

9. Leonard, D. C., Filippenko, A. V., and Chornock, R. T., IAU Circ., No. 7305 (1999). 